\title{
Editorial: Understanding the Growth of the First Supermassive Black Holes
}

\author{
Rosa Valiante ${ }^{1,3}$, Raffaella Schneider ${ }^{1}$ and Marta Volonteri ${ }^{2}$ \\ ${ }^{1}$ INAF - Osservatorio Astronomico di Roma, via di Frascati 33, 00040, Monteporzio Catone, Italy \\ ${ }^{2}$ CNRS, UMR 7095, Institut dAstrophysique de Paris, F-75014, Paris, France \\ ${ }^{3}$ Email: rosa.valiante@oa-roma.inaf.it
}

(Received June 23, 2016; ACCePted June 30, 2016)

The formation, assembly history, and environmental impact of the massive black holes $(\mathrm{BH})$ that are ubiquitous in the nuclei of luminous galaxies today remain some of the main unsolved problems in cosmic structure formation. In the last several years, it has become clear that quasars are not just tracers of early and recent structure formation, but that they seem to have actively influenced galaxies and clusters through feedback mechanisms that are still not well understood. The discovery of more and more numerous quasars at redshift above 6 , powered by BHs with masses similar to that of their local counterparts, further complicates this scenario. This emphasises the urgent need to better understand how and when such massive objects form and grow, what is the strength and scale of their impact on the evolution of their host galaxies, and what are the main physical processes driving and regulating this co-evolution.

The current challenge for theoretical models and numerical simulations is to predict the formation path to the first $z \sim 6$ quasars starting from seed BHs, whilst producing a population in agreement with constraints at lower redshift, including the local Universe. At the same time, observations are tackling the difficult task of detecting the rare, faint, and elusive signatures of the first collapsing and merging BHs, such as the highest redshift emission of gamma-ray bursts, $\mathrm{X}$-rays and, perhaps, gravitational waves.

The papers presented in this special issue are a comprehensive review of the most recent theoretical and observational results presented in July 2015 during the European Week of Astronomy and Space Science (EWASS) Symposium 1. This meeting has been an opportunity to bring together some of leader scientists in the field and a unique opportunity to foster discussion on the relevant processes operating on different scales and in different physical regimes relevant to BHs. In this introduction, we summarise the most recent findings and open questions raised during the conference regarding (i) the formation of $\mathrm{BH}$ seeds and their early growth to the first, high redshift, supermassive BHs (SMBHs); (ii) the physical properties of the first quasars and the co-evolution of their central SMBHs with the host galaxies; (iii) the observational signatures and local relics of SMBHs suggesting strategies/tracers to observe them with the most sensitive and high resolution future instruments.

It has been widely discussed in the literature that if the growth of the first SMBHs starts from BH remnants of Population III (Pop III) stars, i.e. light seeds with mass $\sim 100 \mathrm{M}_{\odot}$, it requires super-Eddington accretion. An alternative route is to start from the so-called heavy $\mathrm{BH}$ seeds, formed by the direct collapse of gas onto a $\sim 10^{5} \mathrm{M}_{\odot}$ BH (e.g. Johnson \& Haardt 2016 for a recent review).

The different channels for BH seeds formation have been presented by several contributors (see the reviews by Volonteri 2010; Latif \& Ferrara 2016), with particular emphasis on the formation mechanisms and relevant processes of the socalled direct collapse scenario, from the formation and mass accretion of a supermassive star (SMS) to its collapse to a massive BH. From reviews and contributed talks, it emerged that direct collapse BHs (DCBHs) of $10^{4}-10^{6} \mathrm{M}_{\odot}$ (e.g. Ferrara et al. 2014), formed by the collapse of gas in massive metal-poor halos exposed to a strong $\mathrm{H}_{2}$ dissociating UV radiation, currently represent the most popular scenario amongst the community working on the first stars. These seeds have been proposed as a viable channel to explain the most luminous quasars $\left(>10^{47} \mathrm{erg} \mathrm{s}^{-1}\right)$ observed at $z>6$ and the cosmic near infrared and X-ray background cross correlation recently detected (Cappelluti et al. 2013).

Andrea Ferrara presented a review on the first stars and $\mathrm{BHs}$ in the reionisation era, discussing the physical relation between stars and $\mathrm{BHs}$ and their role-as sources of UV photons-for reionisation. In his review, Andrea Ferrara stressed that observations of DCBHs would be a key 
discovery to interpret the nature of the cosmic infrared background fluctuations excess and its cross correlation with the X-ray cosmic background. However, the abundance of DCBHs is still uncertain because of the uncertain assumptions adopted to compute their birth mass function, such as the value of the critical intensity of the UV flux required for direct collapse to occur.

A DCBH detection method could be through the detection of narrow $\left(\lesssim 200 \mathrm{~km} \mathrm{~s}^{-1}\right)$ bright Ly $\alpha$ and He II lines, in absence of metal lines. Very bright $\left(>10^{43} \mathrm{erg} \mathrm{s}^{-1}\right) \mathrm{Ly} \alpha$ and $\mathrm{He}$ II narrow lines have been observed in the brightest Ly $\alpha$ emitter, CR7, at redshift $\sim 6.6$ (Matthee et al. 2015; Sobral et al. 2015). It has been proposed that these observed extreme line luminosities can be explained by an accreting DCBH (e.g. Pallottini et al. 2015; Agarwal et al. 2015; Hartwig et al. 2015; Smith, Bromm, \& Loeb 2016; Smidt, Wiggins, \& Johnson 2016). However, a Pop III galaxy nature of this object cannot be ruled out as such extremely bright lines can be also explained through a recent $\lesssim 2$ Myr PopIII star formation episode (Sobral et al. 2015; Visbal, Haiman, \& Bryan 2016; Dijkstra, Gronke, \& Sobral 2016). Unfortunately, clear observational signatures to discriminate amongst the two scenarios (accreting DCBH or Pop III stars) are difficult to identify, as suggested also by other contributors.

Kazuyuki Omukai critically discussed the viability of the DCBHs formation pathway, exploring in detail the environmental conditions that trigger or inhibit gas collapse and the subsequent formation of the SMS: intensity of the UV radiation, gas metallicity, gas monolithic collapse or fragmentation and protostellar (continuous or halted) accretion (Omukai \& Nishi 1999; Omukai 2001; Omukai, Schneider, \& Haiman 2008; Inayoshi \& Omukai 2012; Inayoshi, Omukai, $\&$ Tasker 2014). The formation of a SMS and whether or not it becomes massive enough has been widely investigated by numerical simulations that show that it can occur even in the presence of gas fragmentation. However, UV feedback from the growing star itself can halt the accretion rate and plays a crucial role for the subsequent formation of the massive $\mathrm{BH}$ seed (e.g. Hosokawa, Omukai, \& Yorke 2012; Hosokawa et al. 2013).

There has been a wide consensus that SMS and thus DCBH formation occurs in very rare environments and that the UV background flux illuminating the gas clouds in the protogalaxies plays a crucial role. Muhammad Latif highlighted that one of the key parameters for isothermal direct collapse is in fact, the critical value of the UV flux, $J_{\text {crit }}$, required to suppress the formation of $\mathrm{H}_{2}$. He also presented an alternative scenario for $\mathrm{BH}$ formation in the presence of a moderate amount of $\mathrm{H}_{2}$ cooling. In particular, Latif \& Volonteri (2015) use high-resolution cosmological simulations to study the collapse of massive primordial haloes and the evolution of their dynamical and thermodynamical properties showing that, in some cases, complete isothermal collapse and $\mathrm{H}_{2}$ suppression are not necessary to form a SMS and the large rate required for its efficient accretion $\left(\sim 0.1 \mathrm{M}_{\odot} \mathrm{yr}^{-1}\right)$ can be obtained if the halo is illuminated by an external Lyman
Werner $(\mathrm{LW})$ radiation of $J_{\mathrm{LW}}=(500-1000) 10^{21} \mathrm{erg} \mathrm{cm}^{2} \mathrm{~s}^{1}$ $\mathrm{Hz}^{1} \mathrm{sr}^{1}$ (Latif \& Volonteri 2015).

Different contributors stressed the importance of $\mathrm{H}_{2}$ selfshielding that can prevent the direct collapse favouring gas fragmentation (e.g. Glover 2015a, 2015b and Hartwig et al. 2015), magnetic fields that, on the other hand, can suppress gas fragmentation helping the formation of massive objects (Latif et al. 2013a, 2013b, 2014, 2014a).

The impact of reionisation on the formation of DCBHs has been discussed by Jarrett Johnson who presented cosmological simulations for the collapse of primordial gas into atomic cooling halos, including the effects of both dissociating (LW) and ionising photons. He showed that ionising radiation triggers the formation of Pop III stars thanks to the rapid formation of $\mathrm{H}_{2}$ molecules, favoured by the presence of a large fraction of free electrons in the ionised gas. The subsequent efficient molecular cooling prevents DCBH formation. However, in atomic cooling halos, the dense gas is self-shielded to ionising photons. As a result, photoionisation has only a moderate effect on DCBH formation (Johnson et al. 2012, 2014).

Francesco Haardt reviewed the rate at which seed BHs grow, immediately following their formation. This is dictated both by the mechanical and radiative feedback from their progenitor stars and by the impact of the radiation produced in the accretion process itself. If it occurs from a sufficiently dense gas reservoir, short periods of superEddington accretion represents an efficient mechanism of BH mass growth. This possibility has been the subject of recent studies whose results have been critically discussed (Volonteri, Silk, \& Dubus 2015; Lupi et al. 2016; Pacucci, Volonteri, \& Ferrara 2015; Pezzulli, Valiante, \& Schneider 2016). A third hypothesis has been discussed in this review: The formation of an intermediate mass seed $\mathrm{BH}\left(10^{3}-10^{4}\right.$ $\mathrm{M}_{\odot}$ ), through mergers of stars or stellar mass BHs in dense clusters, may give rise to a SMBH. (e.g. Devecchi \& Volonteri 2009, Devecchi et al. 2012; Lupi et al. 2014).

Recently, Valiante et al. (2016) investigated the role of light and heavy seed BHs in $z>6 \mathrm{SMBHs}$ formation, showing that it strongly depends on the interplay between chemical, radiative, and mechanical feedback effects. They find that Eddington-limited accretion of gas onto few heavy seeds formed at $z>15$ can eventually lead to a $>10^{9} \mathrm{M}_{\odot} \mathrm{BH}$.

One of the important aspects in understanding the formation of the first SMBHs, is the study of the properties of their host galaxies. Observations of quasars at high redshift is the key to unveil the environment in which such objects form and evolve.

Xiaohui Fan summarised the recent results on the physical properties of the most luminous quasars in the early Universe. He presented a new survey at high redshift $(z=5-7)$ based on optical photometry and IR data from UKIDSS and WISE. The new observational techniques allow to expand high-redshift frontiers with more than 200 quasars detected at redshift $z>5$ and $\sim 100$ at redshift $z>6$, powered by SMBHs of more than 10 billion solar masses and shining 
close to the Eddington limit (e.g. Wu et al. 2015). An increasing number of fainter quasars has been observed in the last decade, but the quasar density is exponentially declining at $z>6$, suggesting that we may be running out of $z>6$ quasars. Observations reveal no redshift evolution of the quasar emission and host galaxy metallicity, showing that high-z quasars are old and live in metal-rich environments. However, not all quasars are the same, some of them show very complex gas kinematics that require high sensitivity observations (e.g. with the Atacama Large Millimeter Array, ALMA) to be investigated.

Roberto Maiolino's review focussed on the observational signatures of AGN (negative/positive) feedback at high redshift and implications for BH-host galaxy co-evolution.

It appeared clear from discussions and talks that there is still no consensus on whether $\mathrm{BH}$ growth precedes galaxy assembly or vice versa. Negative feedback has been invoked to prevent the overgrowth of massive galaxies, to explain redand-dead local massive ellipticals and the BH-stellar mass relation, whilst positive feedback has been proposed to explain the observed specific star-formation rate at high redshift, the strong star-formation efficiency of ultra luminous IR galaxies and sub-millimetre galaxies and as an alternative mechanism for the onset of the BH-stellar mass relation. Roberto Maiolino pointed out that observations of gas outflow, traced by $\mathrm{CO}$ and $\mathrm{CII}$ emission lines, suggest that quasar feedback quenching star formation must already be in place in a fraction of massive galaxies at $z \sim 6$, close to the reionisation epoch. However, tracing cold molecular outflows triggered by AGN, at $z>6$, is still very challenging and it has been done only in few bright quasars so far, like SDSSJ1148+5251 in which CO and C II emission lines are spatially resolved, revealing strong and extended emissions correlated to gas outflows $>2000 \mathrm{M}_{\odot} \mathrm{yr}^{-1}$ (Maiolino et al. 2012; Cicone et al. 2015) as expected by theoretical models (e.g. Valiante et al. 2012). However, whether AGN negative feedback can efficiently quench star formation in galaxies is still unclear. Additional mechanisms have been proposed, such as galaxy strangulation, in which infall of cold gas is somehow halted, but is still not clear what processes inhibit gas accretion (Peng, Maiolino, \& Cochrane 2015).

On the other hand, observational evidences of positive feedback suggest that star formation may be triggered by quasar-driven winds at high redshift (e.g. Cresci et al. 2015) suggesting that positive feedback may be an important process in galaxy formation as well. Instruments like ALMA and the James Webb Space Telescope (JWST) will provide major progress on this issue.

Invited and contributed talks indeed underlined that highresolution sub- $\mathrm{mm} / \mathrm{mm}$ observations may lead to a breakthrough in understanding high-z quasar properties, e.g. with instruments like ALMA which is able to spatially resolve C II emission out to redshift $z>6$, enabling the study of complex gas kinematics and rotating discs.

In addition, whilst it is well known that low-J $\mathrm{CO}$ transitions are the best tracer of cold molecular gas in the in- terstellar and intergalactic medium at high redshift, Simona Gallerani discussed how high-J CO transition lines can be used as a tool to detect high-redshift dust-obscured SMBHs ancestors. She reported the first, serendipitous, detection of the $\mathrm{CO}(17-16)$ emission line in the best studied quasar SDSS $\mathrm{J} 1148+5251$ at $z=6.4$ obtained with the Plateau de Bure Interferometer. Models suggest that X-ray dominated regions are required to reproduce such high luminosity line (Gallerani et al. 2014).

Together with high-sensitive, high-resolution observations, theoretical models have a fundamental role in the study of the formation and evolution of the first SMBHs. Tiziana Di Matteo and Yohan Dubois introduced the current advances in numerical simulations aimed to study the rapid growth of BHs at high redshift and their co-evolution with the host galaxy (e.g. Dubois, Volonteri, \& Silk 2014). A large number of semi-analytic models and simulations have been developed to date, with the most updated numerical techniques. In particular, current numerical simulations are growing in size and resolution being able to resolve the full mass function from dwarf galaxies to super clusters of galaxies (e.g. Feng et al. 2015). One of the aims of theoretical models is to study BH-host galaxy scaling relations, such as the BHstellar bulge mass correlation, explaining how and when such relations sets in.

The best observational signatures to constrain the origin of the first SMBHs were discussed during the meeting. The detection of the first massive BHs $\left(10^{5}-10^{7} \mathrm{M}_{\odot}\right)$ at high redshift would provide unique constraints on the $\mathrm{MBH}$ formation mechanism and subsequent growth. Optical, IR, Xray, and radio searches for BHs in dwarf galaxies and the observations of distant $\mathrm{X}$-ray loud and radio galaxies have been proposed as some of the most promising tools by several contributors.

Amy Reines presented ongoing efforts to search for and study the smallest BHs in present-day dwarf galaxies, which are beginning to provide the much needed observational constraints on the masses, host galaxies, and formation path of SMBH seeds. From the first systematic search for AGNs in dwarf galaxies (Reines, Greene, \& Geha 2013), an increasing number of dwarf galaxies with optical signatures of active massive BHs have been identified. These reveal the least massive BHs known so far, $\sim 10^{5}-10^{4} \mathrm{M}_{\odot}$, such as the one in the nucleus of RGG 118 (Baldassare et al. 2015). However, only $\sim 0.5 \%$ of dwarf galaxies present optical signatures of accreting massive $\mathrm{BHs}^{1}$ and thus other diagnostic tools are required, such as X-ray, MIR, and radio observations. The first example of a dwarf starburst galaxy with a massive $\mathrm{BH}$ $\left(\sim 2 \times 10^{6} \mathrm{M}_{\odot}\right)$ has been revealed by means of joint optical $(H S T)$, radio (VLA), and X-ray (CXO) observations in the galaxy Heinze 2-10 (Reines et al. 2011; Reines \& Deller 2012).

\footnotetext{
${ }^{1}$ Such an active occupation fraction translates into a lower limit to the occupation fraction of $\sim 20 \%$ Miller et al. (2015).
} 
Finally, Andrea Comastri emphasised the important role of high energy X-ray observations to reveal the dominant obscured AGN population (more than $50 \%$ of $z>3$ AGN are heavily obscured), presenting the most recent deep XMM and Chandra surveys probing the $z \sim 3-6$ Universe and discussing the expectations and observational strategies for the future surveys with eROSITA and ATHENA Wide Field Imager (WFI). The latter is expected to provide breakthroughs in the determination of the luminosity function and its evolution up to very high redshift ( $z>6$ [Aird et al. 2013]).

As organisers of the EWASS 2015 symposium-entitled Understanding the growth of the first SMBHs-we asked the invited speakers to contribute to this issue with original papers reviewing the results and fruitful discussions presented during the symposium. We thank the EWASS 2015 organisers for giving us the opportunity to hold an interesting meeting in the beautiful island of Tenerife.

\section{REFERENCES}

Agarwal, B., Johnson, J. L., Zackrisson, E., Labbe, I., van den Bosch, F. C., Natarajan, P., \& Khochfar, S. 2016, MNRAS, 460, 4003

Aird, J., et al. 2013, arXiv: 1306.2325

Baldassare, V. F., Reines, A. E., Gallo, E., \& Greene, J. E. 2015 , ApJ, 809, L14

Cappelluti, N., et al. 2013, ApJ, 769, 68

Cicone, C., et al. 2015, A\&A, 574, A14

Cresci, G., et al. 2015, A\&A, 582, A63

Devecchi, B., \& Volonteri, M. 2009, ApJ, 694, 302

Devecchi, B., Volonteri, M., Rossi, E. M., Colpi, M., Portegies Zwart, S. 2012, MNRAS, 421, 1465

Dijkstra, M., Gronke, M., \& Sobral, D. 2016, ApJ, 823, 73

Dubois, Y., Volonteri, M., \& Silk, J. 2014, MNRAS, 440, 1590

Feng, Y., Di Matteo, T., Croft, R., Tenneti, A., Bird, S., Battaglia, N., \& Wilkins, S. 2015, ApJ, 808, L17

Ferrara, A., Salvadori, S., Yue, B., \& Schleicher, D. 2014, MNRAS, 443, 2410

Gallerani, S., Ferrara, A., Neri, R., \& Maiolino, R. 2014, MNRAS, 445, 2848

Glover, S. C. O. 2015a, MNRAS, 451, 2082

Glover, S. C. O. 2015b, MNRAS, 453, 2901

Hartwig, T., Glover, S. C. O., Klessen, R. S., Latif, M. A., \& Volonteri, M. 2015, MNRAS, 452, 1233

Hosokawa, T., Omukai, K., \& Yorke, H. W. 2012, ApJ, 756, 93

Hosokawa, T., Yorke, H. W., Inayoshi, K., Omukai, K., \& Yoshida, N. 2013, ApJ, 778, 178

Inayoshi, K., \& Omukai, K. 2012, MNRAS, 422, 2539

Inayoshi, K., Omukai, K., \& Tasker, E. 2014, MNRAS, 445, L109
Johnson, J. L., \& Haardt, F. 2016, PASA, 33, e007

Johnson, J. L., Whalen, D. J., Agarwal, B., Paardekooper, J.-P., \& Khochfar, S. 2014, MNRAS, 445, 686

Johnson, J. L., Whalen, D. J., Fryer, C. L., \& Li, H. 2012, ApJ, 750, 66

Latif, M. A., Bovino, S., Van Borm, C., Grassi, T., Schleicher, D. R. G., \& Spaans, M. 2014a, MNRAS, 443, 1979

Latif, M. A., \& Ferrara, A. 2016, arXiv: 1605.07391

Latif, M. A., Schleicher, D. R. G., Bovino, S., Grassi, T., \& Spaans M. 2014b, ApJ, 792, 78

Latif, M. A., Schleicher, D. R. G., Schmidt, W., \& Niemeyer, J. 2013a, MNRAS, 433, 1607

Latif, M. A., Schleicher, D. R. G., Schmidt, W., \& Niemeyer, J. C. 2013b, MNRAS, 436, 2989

Latif, M. A., \& Volonteri, M. 2015, MNRAS, 452, 1026

Lupi, A., Colpi, M., Devecchi, B., Galanti, G., \& Volonteri, M. 2014, MNRAS, 442, 3616

Lupi, A., Haardt, F., Dotti, M., Fiacconi, D., Mayer, L., \& Madau, P. 2016, MNRAS, 456, 2993

Maiolino, R., et al. 2012, MNRAS, 425, L66

Matthee, J., Sobral, D., Santos, S., Röttgering, H., Darvish, B., \& Mobasher, B. 2015, MNRAS, 451, 400

Miller, B. P., Gallo, E., Greene, J. E., Kelly, B. C., Treu, T., Woo, J.-H., \& Baldassare, V. 2015, ApJ, 799, 98

Omukai, K. 2001, ApJ, 546, 635

Omukai, K., \& Nishi, R. 1999, ApJ, 518, 64

Omukai, K., Schneider, R., \& Haiman, Z. 2008, ApJ, 686, 801

Pacucci, F., Volonteri, M., \& Ferrara, A. 2015, MNRAS, 452, 1922

Pallottini, A., et al. 2015, MNRAS, 453, 2465

Peng, Y., Maiolino, R., \& Cochrane, R. 2015, Natur, 521, 192

Pezzulli, E., Valiante, R., \& Schneider, R. 2016, MNRAS

Reines, A. E., \& Deller, A. T. 2012, ApJ, 750, L24

Reines, A. E., Greene, J. E., \& Geha, M. 2013, ApJ, 775, 116

Reines, A. E., Sivakoff, G. R., Johnson, K. E., \& Brogan, C. L. 2011, Nature, 470, 66

Smidt, J., Wiggins, B. K., \& Johnson, J. L. 2016, arXiv:1603.00888

Smith, A., Bromm, V., \& Loeb, A. 2016, arXiv:1692.07639

Sobral, D., Matthee, J., Darvish, B., Schaerer, D., Mobasher, B., Röttgering, H. J. A., Santos, S., \& Hemmati, S. 2015, ApJ, 808, 139

Valiante, R., Schneider, R., Maiolino, R., Salvadori, S., \& Bianchi S. 2012, MNRAS, 427, L60

Valiante, R., Schneider, R., Salvadori, S., \& Gallerani, S. 2014, MNRAS, 444, 2442

Valiante, R., Schneider, R., Volonteri, M., \& Omukai, K. 2016, MNRAS, 457, 3356

Visbal, E., Haiman, Z., \& Bryan, G. L. 2016, MNRAS, 460, L59

Volonteri, M. 2010, ARA\&A, 18, 279

Volonteri, M., Silk, J., \& Dubus, G. 2015, ApJ, 804, 148

Wu, X.-B., et al. 2015, Nature, 518, 512 